\title{
H7N9 Influenza A-induced Pneumonia Associated with Acute Myelitis in an Adult
}

\author{
Jun-bo Xia ${ }^{1}$, Jing Zhu ${ }^{2}$, Jun $\mathrm{Hu}^{3}$, Li-min Wang ${ }^{1}$ and Hao Zhang ${ }^{4}$
}

\begin{abstract}
Recently, we encountered three patients infected with an avian influenza A virus (H7N9) who exhibited upper respiratory catarrh symptoms, pharyngalgia, a high fever and hypodynamia in the early stages of the disease. Their conditions deteriorated rapidly, and one-sided pneumonia progressed to two-sided pneumonia. The patients developed respiratory failure and even acute respiratory distress syndrome (ARDS). One patient experienced numbness of the lower limbs, urinary retention and fecal incontinence. Magnetic resonance imaging (MRI) of the spinal cord indicated edema at the thoracic level, and the patient was diagnosed with acute myelitis. This report details the diagnosis and treatment of a patient with H7N9-induced pneumonia associated with acute myelitis.
\end{abstract}

Key words: H7N9, pneumonia, acute myelitis

(Intern Med 53: 1093-1095, 2014)

(DOI: 10.2169/internalmedicine.53.1801)

\section{Introduction}

Since the isolation of a new strain of avian influenza A virus (H7N9) from the pharyngeal secretions of three patients with respiratory tract infections (1), 127 cases and 26 deaths have been reported (2). Patients infected with lowpathogenicity avian influenza A (LPAI) strains, including H7N2, H7N3, H9N2 and H10N7, exhibit conjunctivitis and mild-to-moderate lower respiratory tract signs. Few patients develop acute respiratory distress syndrome (ARDS) (3). The pathogenic H7N9 strain currently prevalent in China can cause severe pneumonia, respiratory failure or even ARDS in some patients (1). However, the mortality from H7N9 is lower than that from the H5N1 strain prevalent in 2003 (4).

Patients infected with influenza seldom display central nervous system symptoms. However, there was a report of acute encephalopathy during the great Spanish flu pandemic of 1918 (5). Recently, there have been several reports of neurologic complications, including posterior reversible encephalopathy syndrome, myelitis, stroke, acute necrotizing encephalitis and seizures (6-8). These complications usually present in the early phase of viral infection or after viral infection and are more prevalent in children. Some healthy people develop acute myelitis following vaccination $(9,10)$. However, the mechanism underlying pathology in the nervous system remains unclear. Of our H7N9-infected patients, one developed numbness of the lower limbs, urinary retention and fecal incontinence. Spinal cord MRI suggested that the patient had acute myelitis. Following the administration of hormone therapy, the patient's condition improved, with a good prognosis. This report describes the treatment and successful outcome of a patient with H7N9-induced pneumonia associated with acute myelitis.

\section{Case Report}

The patient was a 42-year-old man who presented with a fever and cough lasting for three days and was admitted to our hospital. Three days prior to admission, the patient had been exposed to live chickens. One week after that encounter, the patient's body temperature increased to $39.5^{\circ} \mathrm{C}$, and he developed a mild cough, hypodynamia, headache and

\footnotetext{
${ }^{1}$ Department of Pulmonary Medicine, Hangzhou First People's Hospital, China, ${ }^{2}$ Department of Infection Diseases, Hangzhou First People's Hospital, China, ${ }^{3}$ Department of Neurology, the First Affiliated Hospital of the Third Military Medical University Chongqing, China and ${ }^{4}$ Department of Neurology, Hangzhou First People's Hospital, China

Received for publication September 26, 2013; Accepted for publication November 13, 2013

Correspondence to Dr. Jun-bo Xia, xjb47@126.com
} 


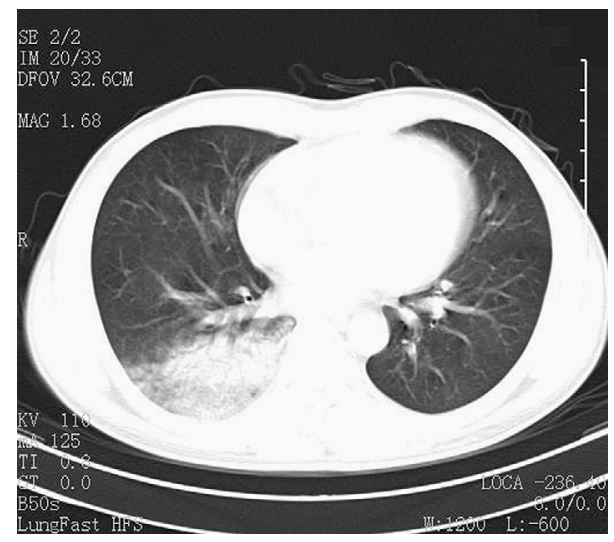

Figure 1. CT scans showed a shadow of consolidation in the lower right lung.

pharyngalgia. A routine blood test conducted at a local hospital showed the following results: white blood cell (WBC) count, $5.6 \times 10^{9} / \mathrm{L}$, neutrophils, $88.1 \%$, leukocytes, $6.9 \%$, and C-reactive protein (CRP), $72 \mathrm{mg} / \mathrm{L}$ (normal range: 0-8 mg/ $\mathrm{L})$. Chest computed tomography (CT) revealed a shadow of consolidation in the lower right lung (Fig. 1). The patient received antibacterial medication (cefuroxime, $2 \mathrm{~g}$ administered intravenously twice a day) for two days.

On the day of admission, the patient visited our fever clinic with new symptoms: numbness of the lower limbs and abdominal distension. His vital signs were as follows: body temperature, $39.8^{\circ} \mathrm{C}$; pulse rate, 96 beats $/ \mathrm{min}$; breath rate, 24 breaths/min; and blood pressure, $126 / 78 \mathrm{mmHg}$. No cyanosis of the lips was observed, and the superficial lymph nodes were not enlarged. The patient had pharyngeal hyperemia, and his tonsils were not enlarged. The trachea was in the middle. Coarse breath sounds were detected over both lungs. Wet rales were heard in the lower right lobe. The cardiac rhythm was stable, without pathologic noise. There was no tenderness, rebound tenderness or muscular tension in the abdomen. The patient was diagnosed with lower-right lung pneumonia.

The patient had limb hypoesthesia and tendon hyporeflexia, and Babinski's reflex was negative. After hospitalization, a repeat blood test showed the following results: WBC count, $5.3 \times 10^{9} / \mathrm{L}$; neutrophils, $80.9 \%$; leukocytes, $16.6 \%$; CRP, $120 \mathrm{mg} / \mathrm{L}$; reticulocytes, 0.29\%; ESR, $74 \mathrm{~mm} / \mathrm{h}$; urinary protein, ++; ALT, $14 \mathrm{U} / \mathrm{L}$; AST, $43 \mathrm{U} / \mathrm{L}$; and LDH, 463 $\mathrm{U} / \mathrm{L}$. The levels of creatinine and urea nitrogen were normal. The results of a blood gas analysis were as follows: $\mathrm{pH}$, 7.43; $\mathrm{PaCO}_{2}, 36 \mathrm{~mm} \mathrm{Hg} ; \mathrm{PaO}_{2}, 54 \mathrm{~mm} \mathrm{Hg} ; \mathrm{HCO}_{3}^{-}, 24$ $\mathrm{mmol} / \mathrm{L} ; \mathrm{SaO}_{2}, 88 \%$. Upon hospitalization, the patient received oxygen via nasal cannula and antibiotic therapy (cefuroxime, $2 \mathrm{~g}$ administered intravenously once a day). By hospital day 3, the numbness of the lower limbs had become aggravated in association with flaccid paralysis, urinary retention, fecal incontinence and worsened dyspnea. The patient lost sensation under the fifth lumbar vertebra. The bilateral muscle strength was grade 0 in the lower extremities, with superficial areflexia. The pathologic characteristics re-

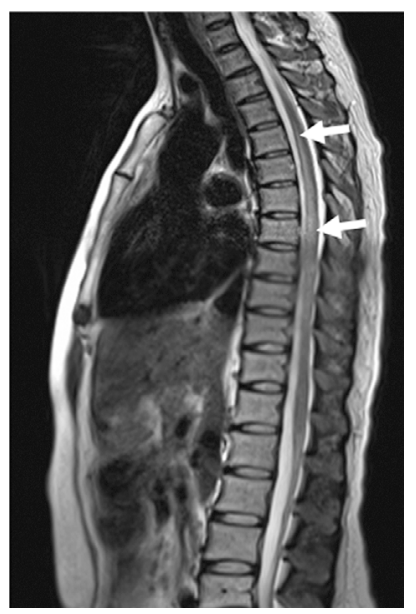

Figure 2. MRI of the spinal cord revealed exudative inflammation in the thoracic cord (arrows).

mained negative. A repeat blood test showed a WBC count of $3.0 \times 10^{9} / \mathrm{L}$, with $82.1 \%$ neutrophils and $12.3 \%$ leukocytes, and a CRP level of $74 \mathrm{mg} / \mathrm{L}$. A reverse transcriptionpolymerase chain reaction (RT-PCR) assay conducted on a throat swab detected H7N9 nucleotides. The pressure of the cerebrospinal fluid was normal, with a protein level of 30 $\mathrm{mg} / \mathrm{L}$ and a WBC count of $8 / \mu \mathrm{L}$ (normal range: $0-5 \mu \mathrm{L}$ ). However, an RT-PCR assay of the cerebrospinal fluid detected no H7N9 nucleotides. Spinal cord magnetic resonance imaging (MRI) suggested the presence of lesions in the thoracic spinal cord; hence, acute myelitis was diagnosed. The results of brain MRI were normal (Fig. 2). Lung CT suggested multiple areas of consolidation in both lungs associated with right pleural effusion (Fig. 3). The patient was diagnosed with H7N9 influenza A-induced pneumonia associated with acute myelitis and received methylprednisolone (40 mg intravenously once a day), daphie (75 mg orally twice a day) and human immunoglobulin ( $\mathrm{pH} 4)(10 \mathrm{~g}$ intravenously once a day). He subsequently recovered and was discharged from the hospital on hospital day 17. Twelve weeks after discharge from the hospital, chest CT was normal. The patient had no discomfort, with a normal respiratory function and blood examination results, and was able to engage in regular physical activities.

\section{Ethics statement}

The study protocol was approved by the ethics review board of the First People's Hospital of Hangzhou. All procedures were performed in accordance with the Declaration of Helsinki and relevant policies in China.

\section{Consent}

Written informed consent was obtained from the patient for publication of this case report and any accompanying images. A copy of the written consent form is available for review from the Editor-in-Chief of this journal. 


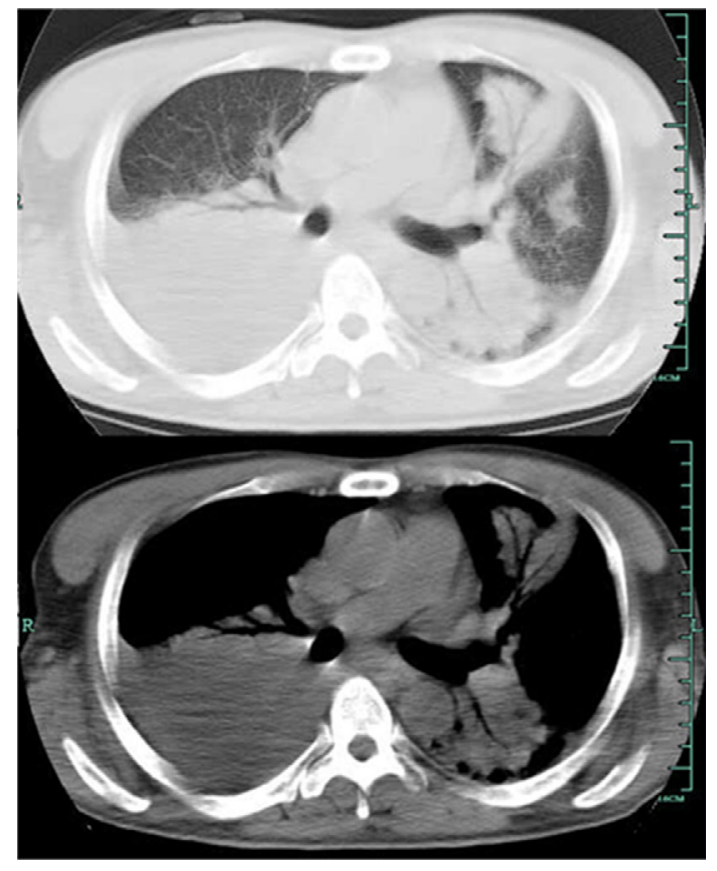

Figure 3. Chest CT demonstrated multiple shadows of consolidation in both lungs on a bronchogram with moderate pleural effusion in the right lung.

\section{Discussion}

The clinical nervous system symptoms and MRI results in this case met the standard criteria for a diagnosis of acute myelitis. Acute myelitis includes Behcet's disease, sarcoidosis, infection (syphilis, Toxoplasma gondii, other viruses), systematic diseases (systematic lupus erythematous, Sjögren's syndrome) and neuromyelitis optica. Respiratory viruses and enteroviruses can also induce acute myelitis. Previous studies have reported that the vaccine for the 2009 subtype of H1N1 influenza A induces acute transverse myelitis $(9,10)$; however, there are no previous reports of influenza A-infected patients with acute myelitis.

At present, the diagnosis of influenza-associated neurologic illness is based on "guilt by association," i.e., if neurologic symptoms are accompanied by acute influenza virus infection of the upper respiratory tract, influenza-associated neurologic illness is diagnosed. In the present patient, the examination of the cerebrospinal fluid showed no specific results or evident inflammation. The detection of viruses in the cerebrospinal fluid and use of the influenza-specific antibody assay is impractical in the clinical setting. Because influenza has no neurotropic characteristics, there is only a low possibility that the development of lesions is directly induced by viral infection. The pathologic changes are more likely caused by an immune response. Neither viral nucleotides nor virus-specific antibodies were detected in the cerebrospinal fluid in this case.

The best method for diagnosing influenza-induced neu- rologic complications is the detection of viral nucleotides or viral replication in upper respiratory tract secretions using RT-PCR. This method is preferred to viral antigen fast testing or immunofluorescence. Therefore, it is important to collect nasopharyngeal specimens as soon as possible if the neurologist is considering a diagnosis of influenzaassociated neurologic disease. The current patient was first diagnosed with pneumonia; however, antibiotic therapy had no effect. His WBC count was low and his lymphocyte level also decreased. His condition deteriorated quickly, and he exhibited nervous system manifestations. We detected viral nucleotides from a throat swab, performed spinal cord MRI and tested the cerebrospinal fluid on the same day. Subsequently, a primary diagnosis of H7N9 influenza A-induced pneumonia associated with acute myelitis was made. The patient then received antiviral and anti-inflammatory therapy and recovered soon thereafter.

The authors state that they have no Conflict of Interest (COI).

\section{Acknowledgement}

This study was funded by the Hangzhou Science and Technology Development Planning Project (Grant No. 20100633B07) and the Hangzhou Medical Science and Technology Development Planning Project (Grant No. 2012A001).

J.B.X. and J.Z. drafted and wrote the manuscript. J.H., L.M. W. and H.Z. were involved in the clinical guidance of the patient and contributed to creating the figures. All authors have read and approved the final manuscript.

\section{References}

1. Gao R, Cao B, Hu Y, et al. Human infection with a novel avianorigin influenza A (H7N9) virus. N Engl J Med 368: 1888-1897, 2013.

2. Chinese Center for Disease Control and Prevention. Available from: URL: http://www.chinacdc.cn/jkzt/crb/rgrgzbxqlg_5295/ rgrqlgyp/201304/t20130408_79589.htm

3. Fouchier RA, Schneeberger PM, Rozendaal FW, et al. Avian influenza Avirus (H7N7) associated with human conjunctivitis and a fatal case of acute respiratory distress syndrome. Proc Natl Acad Sci U S A 101: 1356-1361, 2004.

4. Nicholson KG, Wood JM, Zambon M. Influenza. Lancet 362 : 1733-1745, 2003.

5. Neurological complications of influenza. BMJ 1: 248-249, 1970.

6. Toovey S. Influenza-associated central nervous system dysfunction: a literature review. Travel Med Infect Dis 6: 114-124, 2008.

7. Maricich SM, Neul JL, Lotze TE, et al. Neurologic complications associated with influenza A in children during the 2003-2004 influenza season in Houston, Texas. Pediatrics 114: e626-e633, 2004.

8. Bartynski WS, Upadhyaya AR, Boardman JF. Posterior reversible encephalopathy syndrome and cerebral vasculopathy associated with influenza A infection: report of a case and review of the literature. J Comput Assist Tomogr 33: 917-922, 2009.

9. Gui L, Chen K, Zhang Y. Acute transverse myelitis following vaccination against $\mathrm{H} 1 \mathrm{~N} 1$ influenza: a case report. Int J Clin Exp Pathol 4: 312-314, 2011.

10. Korn-Lubetzki I, Dano M, Raveh D. H1N1 vaccine-related acute transverse myelitis. Isr Med Assoc J 13: 249-250, 2011.

(C) 2014 The Japanese Society of Internal Medicine http://www.naika.or.jp/imonline/index.html 